Research Article,

\title{
A Study of Anatomical and Visual Outcome in Secondary Scleral Fixated Intra-Ocular Lens Implantation
}

\author{
Dr Sachin Agrawal ${ }^{1}$, Dr Ravi Chauhan², Dr Sagar Rajurkar ${ }^{3}$ \\ Indira Gandhi Government Medical College \& Hospital, Nagpur India \\ Email Address: sagar25rajurkar@gmail.com
}

\begin{abstract}
:
Aims: To study of anatomical and visual outcome in secondary scleral fixated intra-ocular lens implantation Settings and Design: Hospital based prospective case study conducted at a tertiary care centre over a period of 2 years.

Methods and Material: 30 eyes of 30 patients underwent secondary scleral fixation of IOL by 2-point Abexterno technique. Post-operatively best corrected visual acuity, IOP, Keratometry and complications encountered were noted at regular follow-up of 7th day, 6 weeks, 3 months and 6 months.

Results: Mean age of patients in the study was 52.33 years in the range of $46-60$ years. Out of the 30 cases, $56.66 \%$ were men and $43.33 \%$ were women. Best corrected pre-operative V/A was in the range of $6 / 24$ to $6 / 18(53.33 \%), 6 / 12$ to $6 / 9$ (16.66\%), $6 / 60$ to $6 / 36(30 \%)$. At final follow-up of 6 months, the results were, $63.33 \%$ in range of $6 / 12$ to $6 / 9,26.66 \%$ in $6 / 24$ to $6 / 18$ and $10 \%$ in $6 / 60$ to $6 / 36$. In early complications, transient corneal oedema was seen in $20 \%$, transient IOP elevation in $10 \%$, while vitreous haemorrhage and post-operative hyphaema was noted in $3.33 \%$ each. In late complications, Cystoid Macular Oedema was seen in $10 \%$, pupil distortion was seen in $6.66 \%$ of patients and decentred IOL was seen in $3.33 \%$ of the patients.

Conclusion: Secondary scleral fixated IOL provides good visual outcome and better anatomical position with minimal complications.
\end{abstract}

Keywords: Secondary scleral fixated IOL, Visual outcome, Anatomical outcome.

\section{Introduction:}

In the modern era of phacoemulsification and improved surgical instrumentation, cataract surgery has become one of the safest and gratifying surgical procedure with extreme expectations on part of the patient. Patients want a spectacle free perfect post-operative outcome. There are a variety of cataract extraction methods, including phacoemulsification, extracapsular cataract extraction and intracapsular cataract extraction. In intracapsular cataract extraction (ICCE), the lens is removed along with the capsule and in Extracapsular cataract extraction (ECCE), posterior capsule is spared. Since 1970, phacoemulsification and extracapsular cataract surgery have replaced intracapsular cataract surgery.
Modern cataract surgery involves phacoemulsification of the opacified crystalline lens and the implant of an intraocular lens (IOL) in the capsular bag. In some conditions this is not possible due to the type of cataract (e.g., traumatic cataracts with lens subluxation, cataracts in pseudo exfoliative syndrome with zonular/capsular dehiscence) or to systemic and congenital disorders characterized by weakness of zonules/capsule (e.g., familial or idiopathic ectopia lentis, Marfan syndrome, etc.) or to intraoperative complications (e.g., large breaks of the posterior capsule, accidental aspiration of the capsular bag, etc.) leaving the patient aphakic. ${ }^{[1]}$

Aphakia can be corrected by either spectacle lens, contact lens or intraocular lens. The insertion of an intraocular lens within the aphakic eye overcomes 
the optical disadvantages of aphakic spectacles and the handling and wearing difficulties encountered with contact lens. ${ }^{[2,3]}$ An IOL can be placed in the anterior chamber between the cornea and iris, as in iris-fixated and closed- or open-loop anterior chamber IOLs (AC-IOLs), or it can be implanted in the posterior chamber within the ciliary sulcus posterior to the iris, as in sutured iris-fixated and sclera fixated posterior chamber IOLs (PC-IOLs). Out of all these techniques, Scleral fixated PCIOL implantation has several advantages over other secondary IOLs. Because of its normal physiological location, SF-IOL has less chances of corneal endothelial decompensation, chronic inflammation, peripheral anterior synechiae, glaucoma, shallow anterior chamber, or cystoid macular oedema and less trauma to anterior chamber structures.

In this study, we analyse the indications, safety, efficacy, postoperative outcome, and complication rate in cases where secondary scleral fixated PCIOL implantation was done.

\section{Materials and Methods:}

This was a hospital based prospective clinical trial done in tertiary care centre setup. We studied the secondary scleral fixated intra-ocular lens implantation in 30 patients who fulfilled inclusion criteria over a period of 2 years. Patients included in this study were the Post cataract surgery aphakic patients with deficient posterior capsular support (with or without disturbed anterior vitreous phase), and post cataract surgery aphakic patients in case of grossly Subluxated lens. Following patients were excluded from the study, Patients having No PL \& defective PR in traumatic cataract, Patients with causes of poor vision other than cataract, Subluxated cataract of less than 7 clock hours, Post-traumatic corneal opacity in optical zone, Traumatic cataract with angle recession, Patients with traumatic posterior lens dislocation and Traumatic cataract with retained intra-ocular foreign bodies.

All patients were thoroughly investigated and underwent secondary scleral fixation of IOL by 2point Ab-externo technique by the same surgeon. Single piece polymethyl methacrylate (PMMA) with modified $\mathrm{C}$ haptics with at least two dialing holes in the haptics, with an optical diameter of at least $6.5 \mathrm{~mm}$, with overall diameter of $13.5 \mathrm{~mm}$ was used. Post-operatively best corrected visual acuity, IOP, keratometry and complications encountered were noted at regular follow-up of 7 days, 6 weeks, 3 months and 6 months. Data on subsequent follow up was gathered and analysed.

\section{Results:}

Maximum numbers of patients were in the age group of 46-60 years (63.33\%) whereas, least cases were found in 16-30 years of age group. Mean age of patients in our study was 52.33 years in the range of 46-60 years. Minimum age was 26 years of age and maximum was 72 years of age. (Table no.1)

Out of the 30 cases, $56.66 \%$ were men and $43.33 \%$ were women, thus in this study men dominated with M:F ratio of 1.31: 1. (Table no.2)

Secondary scleral fixation of PC-IOL was done for the aphakic patients who presented to our OPD. $20 \%$ of the patients were aphakic after the removal of grossly subluxated lens and $80 \%$ of the patients were aphakic after the cataract extraction resulting in posterior capsular rupture or deficient posterior capsule. The mean time interval between the cataract extraction and secondary SFIOL implantation was 3 weeks. (Table no.3)

Pre-operatively, the visual acuity was meticulously measured, so as to compare the post-operative BCVA after secondary implantation of SFIOL. Most of our patients had best corrected preoperative V/A in the range of $6 / 24$ to $6 / 18$ $(53.33 \%) .16 .66 \%(n=5)$ patients had BCVA of $6 / 12$ to $6 / 9$ while $30 \%(n=9)$ patients had visual acuity $6 / 60$ to $6 / 36$. (Table no.4) Visual acuity was checked with pin-hole on Snellen Chart on the immediate post-operative day. Of the total patients, $23.33 \%(n=7)$ of our patients had visual acuity of $6 / 12-6 / 9$, another $43.33 \%(n=13)$ had it in the range of $6 / 24-6 / 18$ while $23.33 \%(n=7)$ had visual acuity in the range of $6 / 60-6 / 36$. The remaining $10 \%$ had visual acuity of less than $6 / 60$ on $1^{\text {st }}$ postoperative day.

Post-operatively visual acuity was checked at 1 week, $23.33 \%$ of our patients had BCVA in the range of $6 / 60$ to $6 / 36,43.33 \%$ of patients had BCVA of $6 / 24$ to $6 / 18$ and the remaining $33.33 \%$ of the patients had vision in the range of $6 / 12$ to 6/9. Post-operatively V/A was checked on Snellen chart at 6 weeks. $43.33 \%$ of the patients had BCVA in the range of $6 / 12$ to $6 / 9$. $40 \%$ of the patients had it in the range of $6 / 24$ to $6 / 18$ and remaining $16.66 \%$ of cases had BCVA of $6 / 60$ to $6 / 36$.

At 3 months post-operatively, patients with BCVA in the range of $6 / 12$ to $6 / 9$ increased to $63.33 \%$ while $10 \%$ of patients had BCVA in the range of $6 / 60$ to $6 / 36$ and $26.66 \%$ of patients had BCVA of 
6/24 to 6/18. (Table no.5)

Pre-operatively all the patients were having intraocular pressure $<21 \mathrm{~mm}$ hg. At Post-operative follow-up at 1 st week, $90 \%$ of the patients had intra-ocular pressure in the normal range i.e., $<21$ $\mathrm{mm}$ hg, while $10 \%$ of the patients had raised IOP. At the end of the study i.e. at 6 months postoperatively, all the patients were having IOP in the normal range. Pre-operatively every patient was checked for corneal astigmatism by keratometry. It was seen that $46.66 \%$ of the patients had astigmatism of $<1 \mathrm{D}$ while $53.33 \%$ of the patients had astigmatism of more than $1 \mathrm{D}$ as all these patients had undergone primary cataract extraction prior to the secondary implantation of SF-PCIOL. Post-operatively on keratometric examination, at 6 months, we found $66.66 \%$ of our patients had astigmatism in the range of 1.0 to $2.5 \mathrm{D}$. In early complications, transient corneal oedema was seen in $20 \%$ of patients, transient IOP elevation was seen in $10 \%$, while vitreous haemorrhage and postoperative hyphaema was noted in $3.33 \%$ each. In late complications, Cystoid Macular Oedema was seen in $10 \%$ of the patients, pupil distortion was seen in $6.66 \%$ of patients and decentred IOL was seen in $3.33 \%$ of the patients. Vision threatening complications like Retinal Detachment and Endophthalmitis were not seen in any patients. (Table no.6)

\section{Discussion:}

In the present prospective clinical trial, we studied the anatomical and visual outcome in secondary scleral fixated intraocular lens implantation. The study was conducted over a period of 24 months from November 2017 to October 2019. We studied the results of secondary scleral fixation of IOL in 30 cases.

Though, implantation of modern, flexible, open loop AC-IOLs has regained the popularity, they are still associated with variety of complications such as corneal oedema, peripheral anterior synechiae, secondary glaucoma, uveitis, cystoid macular oedema and hyphaema. Similarly, iris fixated PCIOLs are associated with various iris related complications like iritis, iris pigment dispersion, glaucoma and iris neovascularization. ${ }^{[5]}$ On other hand, scleral fixated PCIOL implantation technique is still evolving. It offers good visual outcome with relatively low complication rate and gives good anatomical results. The advantages of scleral fixated PCIOL implantation. ${ }^{[6]}$ are that they preserve the corneal endothelial cells, reduce risk of injury to iris and ciliary body and produce stable, long term fixation. In addition, it is implanted in the same plane as the crystalline lens, therefore it is optically more physiological.

Scleral fixation of the PCIOLs can be done by different techniques which include the Ab-externo technique and Ab-interno technique. Nikita Gabric et $a .^{[4]}$ studied the complications of both the techniques in details and found that the complications rates were more and visual outcome less favourable with $\mathrm{Ab}$-interno scleral fixation technique. In our study of secondary implantation of SF-PCIOL with anterior vitrectomy, whenever indicated was done. The technique used in the study was Ab-externo 2-point scleral fixation technique. All the patients were operated by the same surgeon. They were meticulously followed up for up to 6 months and the results were documented. $20 \%$ of the patients were aphakic after the removal of grossly subluxated lens and $80 \%$ of the patients were aphakic after the cataract extraction resulting in posterior capsular rupture or deficient posterior capsule. The mean time interval between the cataract extraction and secondary SFIOL implantation was 3 weeks. At our final follow-up of 6 months, the results were similar to 3 months with $63.33 \%$ of patients having BCVA in the range of $6 / 12$ to $6 / 9,26.66 \%$ of patients in the range of $6 / 24$ to $6 / 18$ and $10 \%$ of patients in the range of $6 / 60$ to $6 / 36$.

Zuleyha Yalniz-Akkaya, et. al., ${ }^{[8]}$ studied the secondary scleral fixated IOLs in a total of 39 eyes who were aphakic at the time of surgery, with a median aphakic period of 5 months. Thirty aphakic eyes $(50.8 \%)$ had previous complicated phacoemulsification related to posterior capsule rupture or zonular dialyses, 4 aphakic $(6.8 \%)$ eyes had previous uncomplicated cataract surgery due to congenital cataract and 5 eyes $(8.5 \%)$ had previous reoperation due to the penetrating ocular injury. Seven eyes $(11.9 \%)$ had ACIOL-related complications and 13 eyes $(22 \%)$ had IOL dislocation. Santosh Kumar, et al. ${ }^{[1]} 32$ patients undergo secondary SFIOL implantation, majority of patients had best corrected pre-operative visual acuity in the range of $6 / 36$ to $6 / 24$. Dadeya S., et al. ${ }^{[9]}$ studied the secondary implantation of SF-IOL in 32 patients. Best corrected pre-operative visual acuity was $<6 / 60$ in 5 patients $(15.6 \%), 6 / 60$ in 5 patients $(15.6 \%), 6 / 36$ in 8 patients $(25 \%)$ and $6 / 24$ in 14 patients $(43.8 \%)$. Lee V.Y.W. et al. ${ }^{[7]}$ got BCVA in group of secondary placements of SFIOL of $6 / 12$ or better in $19(76.0 \%)$ of cases, 6 (24\%) cases got $6 / 15$ to $6 / 60 \mathrm{BCVA}$ at the final 
follow-up of 17.5 months. S. Dadeya, et al. ${ }^{[9]}$ found BCVA of $6 / 18$ or better in $30 \%$ of patients who underwent secondary SFIOL after mean follow-up of 3 years. Uthoff D, et al. ${ }^{[10]}$ got BCVA improved or unchanged after secondary IOL implantation with SFIOL in $92 \%$ of eyes.

In early complications, transient corneal oedema was seen in $20 \%$ of patients, transient IOP elevation was seen in $10 \%$, while vitreous haemorrhage and post-operative hyphaema was noted in $3.33 \%$ each. In late complications, Cystoid Macular Oedema was seen in $10 \%$ of the patients, pupil distortion was seen in $6.66 \%$ of patients and decentred IOL was seen in $3.33 \%$ of the patients. Vision threatening complications like Retinal Detachment and Endophthalmitis were not seen in any patients.

Lee V.Y.W. et al. ${ }^{[7]}$ in his study of comparison between primary and statistically significant incidence of corneal oedema $(44 \%)(n=11)$ in patients undergoing secondary scleral fixation and attributed reduced BCVA to it. Muhittin Taskapilli et al. ${ }^{[14]}$ reported corneal oedema in $30 \%$ cases. Corneal oedema in $20 \%$ cases of our study is much less than that reported by other authors. Lee V.Y.W. et al., ${ }^{[7]}$ Chan C.C. et al. and Gondula B. et al. reported transient rise in IOP during early postoperative period in $28 \%, 17 \%$ and $30 \%$ cases respectively. We got CME in $10 \%$ of our cases. When compared to other studies we found that CME was leading cause of reduced BCVA in many of the studies. Nikita Gabric et al. ${ }^{[4]}$ documented CME in $16 \%$ cases, Lanzetta P. et al. $^{[12]}$ found CME in $30 \%$ cases while Chakrabarti A. et al. ${ }^{(13)}$ reported CME in $12 \%$ cases. On other hand ZuleyhaYalniz-Akkaya et al. ${ }^{[8]}$ reported CME in secondary scleral fixation in $4(6.8 \%)$ of patients. Uthoff D. et al. ${ }^{[10]}$ reported CME in $5.8 \%$ of cases. Our results were comparable to these studies. Hayashi K. et al. ${ }^{[15]}$ found that both IOL tilt or decentration were more common with the scleral fixated IOL than with the "in the bag" or "out of the bag" fixated IOLs. Holladay J.T. ${ }^{[16]}$ found that decentration greater than $1.0 \mathrm{~mm}$ causes radial astigmatism and tilt greater than 15 degrees causes coma aberration that cannot be fully corrected with spectacles. Kaynak S. et al. ${ }^{[17]}$ reported neither tilt nor decentration in their series. Lee V.Y.W. et al. ${ }^{[7]}$ reported one case in his study.

\section{Conclusion:}

Secondary scleral fixation of PCIOLs gives good visual outcome in aphakic patients with deficient posterior capsular support. The anatomical position of IOL in secondary SF-PCIOL implantation is well maintained by this technique. Minor complications are common; however, vision threatening complications are rare after secondary SF-PCIOL implantation. Technique is difficult to master, but is generally safe \& effective on short term follow-up.

\section{References:}

[1] Cellini M, Strobbe E, Toschi PG, et al. Secondary IOL Implantation without Capsular Support: A Laser Flare Cell Meter Study; ISRN Ophthalmology 2011; 1-5

[2] Elkington AR, Frank HJ, Greaney MJ. Optics of ametropia. Clinical optics. 3 rd ed. Blackwell science 2005:113-140

[3] Hayward JM, Noble BA, George N. Secondary Intraocular Lens Implantation: Eight Year Experience. Eye 1990; 4, 548556

[4] Gabrić N, Henč-Petrinović L, Dekaris I. Complications following two methods of posterior chamber intraocular lens suturing. Documenta 1996;92(2):107-116.

[5] Chan C.C., Crandall A.S., Ahmed I.I. Abexterno scleral suture loop fixation for PCIOL decentration: clinical results. Jr. Cataract refractive surgery. 2006 Jan; 32(1), 121-8.

[6] Evereklioglu C., Er H., Bekir N., Borazan M., Zorlu F. Comparison of secondary implantation of flexible open-loop anterior chamber and scleral-fixated posterior chamber intraocular lenses. Journal of Cataract \& Refractive Surgery. 2003;29(2):301-308.

[7] Lee V.Y.W., Yuen H.K., Kwok A.K. Complication of outcome of primary and secondary implantation of SFPCIOL. British J Ophthalmol. 2003 Dec; 87(12): 1459-62.

[8] Yalniz-Akkaya Z, Burcu A, Uney GO, Abay I, Eksioglu U, Acar MA, Ornek F. Primary and secondary implantation of scleral-fixated posterior chamber 
intraocular lenses in adult patients. Middle

East Afr J Ophthalmol 2014;21:44-9

[9] Dadeya S., Kamlesh K., Sodhi P. Secondary IOL implantation: Anterior chamber vs scleral fixation long term comparative evaluation. Eur J Ophthalmol. 2003 AugSept.; 13(7): 627-33.

[10] Uthoff D., Teichman K.D. Secondary implantation of SFIOL. Jr. Cataract Refract Surg. 1998 July; 24(7): 945-50

[11] Kumar S, Singh S, Singh G, Rajwade NS, Bhalerao SA, Singh V. Visual outcome and complications of various techniques of secondary intraocular lens. Oman $J$ Ophthalmol. 2017;10(3):198-204. doi:10.4103/ojo.OJO_134_2016

[12] Lanzetta P., Menchini U., Virzili G. Scleral fixated IOL : An angiographic study. Retina. 1998 Jan; 18(6): 515-20.

[13] Chakrabarti A., Gandhi R.K., Chakrabarti M. Ab-externo 4-point scleral fixation of posterior chamber IOLs. J. Cataract refractive Surgery. 2006 Jan;32(1),121-8.

[14] Yalniz-Akkaya Z, Burcu A, Uney GO, Abay I, Eksioglu U, Acar MA, Ornek F. Primary and secondary implantation of scleral-fixated posterior chamber intraocular lenses in adult patients. Middle East Afr J Ophthalmol 2014;21:44-9

[15] Hayashi K., Hayashi. H, Nakao F. Intraocular lens tilt and decentration, anterior chamber depth and refractive error after trans-scleral suture fixation surgery. Ophthalmology 1999; 878-82.

[16] Holladay J. T. Evaluating the intraocular lens optics. Sur. Ophthalmol. 1986; 30: 385-90.

[17] Kaynak S., Ozbek Z., Pasa E., Oner F. H., Cingil G. Trans-scleral fixation of foldable intraocular lenses. J cataract refract surg. 2004; 30: 854-7. 